\title{
Hamiltonian Formalism of Whitham-Type Hierarchies and Topological Landau-Ginsburg Models
}

\author{
B. A. Dubrovin * \\ Department Mechanics and Mathematics, Moscow State University, SU-119899 Moscow, USSR
}

Received August 15, 1991

\begin{abstract}
We show that the bi-hamiltonian structure of the averaged Gelfand-Dikii hierarchy is involved in the Landau-Ginsburg topological models (for $A_{n}$-Series): the Casimirs for the first P.B. give the correct coupling parameters for the perturbed topological minimal model; the correspondence \{coupling parameters $\} \rightarrow$ primary fields $\}$ is determined by the second P.B. The partition function (at the tree level) and the chiral algebra for LG models are calculated for any genus $g$.
\end{abstract}

\section{Introduction}

We start with explanation of the term "Whitham-type hierarchy" and with brief summary of the Landau-Ginsburg potential formalism in topological minimal models.

Whitham-type hierarchy. Let

$$
\partial_{t_{a}} \psi=F_{a}\left(\psi, \psi_{x}, \ldots\right), \quad a=1,2, \ldots
$$

be a KdV-type hierarchy of pairwise commuting evolutionary systems. Let us fix a $N$-dimensional family of invariant $m$-tori. In other words we fix a family of exact solutions of $(0.1)$ of the form

$$
\psi=\Psi\left(t_{1} \kappa^{(1)}+t_{2} \kappa^{(2)}+\cdots+\varphi^{0} ; u^{1}, \ldots, u^{N}\right)
$$

(let $F_{1}=\psi_{x}$ so $\left.t_{1} \equiv x\right)$. Here $\Psi=\Psi\left(\varphi_{1}, \ldots, \varphi_{m} ; u^{1}, \ldots, u^{N}\right)$ is a $2 \pi$-periodic in $\varphi_{1}, \ldots, \varphi_{m}$ function depending on the parameters $u=\left(u^{1}, \ldots, u^{N}\right)$; $\kappa^{(a)}=\left(\kappa_{1}^{(a)}(u), \ldots, \kappa_{m}^{(a)}(u)\right) ; \varphi^{0}=\left(\varphi_{1}^{0}, \ldots, \varphi_{m}^{0}\right)$ is an arbitrary phase shift. The parameters $u=\left(u^{1}, \ldots, u^{N}\right)$ belong to a $N$-dimensional manifold $M$. In the nonlinear WKB-approximation [1] ("Whitham averaging method") the hierarchy $(0.2)$

* Address for 1991/1992 acad. year: Universitá degli Studi di Napoli, Dipartimento di Scienze Fisiche - Mostra d'Oltremare, Pad. 19, I-80125 Napoli, Italy. Fax (39) 81-7253449 
in a vicinity of the invariant submanifold (0.2) can be described by an "averaged hierarchy" of dynamical systems on the loop space $\mathscr{L} M$ of the form

$$
\partial_{T_{a}} u^{i}=\sum_{j=1}^{N} v_{\jmath, a}^{i}(u) u_{X}^{j} .
$$

Here $X=\varepsilon x, T_{a}=\varepsilon t_{a}$ are the "slow variables," $\varepsilon$ is a small parameter. Though all the systems $(0.3)$ commute pairwise the averaged hierarchy $(0.3)$ is not complete (for $m>0)$. The completion of it of the form

$$
\partial_{T_{A}} u^{i}=\sum_{j=1}^{N} v_{j, A}^{i}(u) u_{X}^{j}, \quad \partial_{T_{A}} \partial_{T_{B}}=\partial_{T_{B}} \partial_{T_{A}}
$$

we shall call a Whitham-type hierarchy. An example of the Whitham-type hierarchy being obtained via the above procedure of averaging and extension from the GelfandDikii hierarchy will be given below (the more general case was considered in [2]).

For a wide class of local Hamiltonian structures

$$
\left\{\psi^{p}(x), \psi^{q}(y)\right\}=\sum_{k} B_{k}^{p q}\left(\psi(x), \psi_{x}(x), \ldots\right) \delta^{(k)}(x-y)
$$

of $(0.1)$,

$$
F_{a}=\left\{\psi(x), H_{a}\right\}, \quad H_{a}=\int P_{a}\left(\psi, \psi_{x}, \ldots\right) d x,
$$

the averaging procedure for the Poisson brackets $\{$,$\} was proposed in [3] (see also$ [4]),

$$
\begin{gathered}
\{,\} \stackrel{\text { averaging }}{\longrightarrow}\{,\}_{\mathrm{aver}} \cdot \\
\left\{u^{\imath}(X), u^{j}(Y)\right\}_{\mathrm{aver}}=g^{i s}(u(X))\left[\delta_{s}^{j} \delta^{\prime}(X-Y)-\Gamma_{s k}^{\jmath}(u) u_{X}^{k} \delta(X-Y)\right], \\
v_{j, a}^{i}(u) u_{X}^{j}=\left\{u^{i}(X), H_{a}^{\text {aver }}\right\}_{\mathrm{aver}}, \\
H_{a}^{\mathrm{aver}}=\int \bar{P}_{a}(u(X)) d X, \quad \bar{P}_{a}(u)=(2 \pi)^{-m} \oint P_{a}(\Psi(\varphi, u), \ldots) d^{m} \varphi .
\end{gathered}
$$

("P.B. of hydrodynamic type"). Here $g^{\imath j}(u)$ are the contravariant components of some metric on $M$ and $\left(\Gamma_{j k}^{2}(u)\right)$ is the corresponding Levi-Cività connection. It follows from the general theory of P.B. of hydrodynamic type $[3,4]$ that the metric $g^{i j}(u)$ is flat. In the flat co-ordinates $v^{\alpha}=v^{\alpha}(u), \alpha=1, \ldots, N$, the P.B. (0.8) has a constant form

$$
\left\{v^{\alpha}(X), v^{\beta}(Y)\right\}_{\mathrm{aver}}=\eta^{\alpha \beta} \delta^{\prime}(X-Y)
$$

for some constant symmetric matrix $\eta^{\alpha \beta}$. In other words the flat co-ordinates for the metric $g^{i j}$ are the Darboux-type co-ordinates for the P.B. (0.8). The functionals

$$
\int v^{\alpha} d X, \quad \alpha=1, \ldots, N
$$

are the Casimirs of $(0.8)$. The flat co-ordinates $v^{\alpha}$ can be described in terms of the original P.B. (0.5) as follows [3]: they are the Casimirs for $\{$,$\} , action variables$ $J_{1}, \ldots, J_{m}$ (for $(0.5)$ on the tori $(0.2)$ ) and the wave numbers $\kappa_{1}^{(1)}, \ldots, \kappa_{m}^{(1)}$.

Topological Landau-Ginsburg Models. We shall discuss here neither the definition of topological conformal field theory models nor the construction of these models by twisting of $N=2$ superconformal field theories [5]. But we give a summary of some 
important properties of these theories. The main features of a topological field theory are the following points.

1) All the correlation functions do not depend on co-ordinates.

2) All of them can be expressed via correlators of primary fields $\Phi_{1}, \ldots, \Phi_{N}$.

3) Factorization rules: let \langle\rangle denote tree-level correlators,

$$
\begin{gathered}
\eta_{\alpha \beta}=\left\langle\Phi_{\alpha} \Phi_{\beta}\right\rangle, \quad \operatorname{det}\left(\eta_{\alpha \beta}\right) \neq 0 \\
C_{\alpha \beta \gamma}=\left\langle\Phi_{\alpha} \Phi_{\beta} \Phi_{\gamma}\right\rangle .
\end{gathered}
$$

Then

$$
\left\langle\Phi_{\alpha} \Phi_{\beta} \Phi_{\gamma} \Phi_{\delta}\right\rangle=C_{\alpha \beta}^{\varepsilon} C_{\varepsilon \gamma \delta}
$$

etc. Here

$$
C_{\alpha \beta}^{\varepsilon}=C_{\alpha \beta \mu} \eta^{\mu \varepsilon}, \quad\left(\eta^{\alpha \beta}\right)=\left(\eta_{\alpha \beta}\right)^{-1}
$$

In turns out that $C_{\beta \gamma}^{\alpha}$ are structure constants of a commutative associative algebra $A$ coinciding with the chiral ring of the primary fields. The double-point correlators provide an invariant scalar product $\langle$,$\rangle on A,\langle a b, c\rangle=\langle a, b c\rangle$. Also the algebra $A$ has a unit $\Phi_{1}$ such that

$$
\left\langle\Phi_{1} \Phi_{\alpha} \Phi_{\beta}\right\rangle=\eta_{\alpha \beta}
$$

We recall [6] that such an algebra $A$ is called (commutative) Frobenius algebra.

4) One should consider a family of perturbed topological models depending on $N$ coupling parameters. The corresponding coupling space $M$ should carry an affine structure with marked direction $t_{1}$. A 1-1 correspondence

$$
\{\text { coupling space }\} \leftrightarrow\{\text { primary fields }\}
$$

should be fixed. In affine co-ordinates $t_{1}, \ldots, t_{N}$ on the coupling space one has

$$
t_{1}, \ldots, t_{N} \leftrightarrow \Phi_{1}, \ldots, \Phi_{N}
$$

All the correlators are functions on the coupling space. The main feature of the identification (0.16) is in formulae of the form

$$
\left\langle\Phi_{\alpha} \Phi_{\beta} \Phi_{\gamma} \int \Phi_{\delta}\right\rangle=\partial_{t_{\delta}} C_{\alpha \beta \gamma}
$$

Also one has

$$
\partial_{t_{\gamma}} \eta_{\alpha \beta} \equiv 0
$$

This provides a structure of (complex) Euclidean space in the coupling space $M$.

5) For the (logarythm of) partition function $F=F(t)$ of the perturbed topological theory (at the tree level) the following identity holds

$$
\partial_{t_{\alpha}} \partial_{t_{\beta}} \partial_{t_{\gamma}} F(t)=C_{\alpha \beta \gamma}(t)
$$

From $(0.15),(0.19)$ one has also

$$
\partial_{t_{1}} \partial_{t_{\alpha}} \partial_{t_{\beta}} F(t)=\eta_{\alpha \beta}
$$

We obtain therefore the following problem of "nonlinear equations theory": how to describe $N$-parameter deformations $C_{\alpha \beta}^{\gamma}(t)$ of $N$-dimensional Frobenius algebra being representable in the form $(0.20),(0.21),(0.14)$. Here we construct a "genus $g$ " solution of this problem using the Landau-Ginsburg (LG) potentials approach being proposed in [7] for genus 0 . That means that the partition function $F$ having been constructed is defined on an appropriate moduli space of algebraic curves of genus 
g. In the Appendix we give another class of deformations using the geometry [9] of Frobenius algebras.

The LG potentials machinery was used for calculating the deformations of the chiral algebra in [7] for the genus zero case (the equivalence of this approach to the $N=2$ superconformal field theory approach was considered in [8]). We give here the LG formulae for $A_{n-1}$-models only. The coupling space is the family of polynomials

$$
M=\left\{\lambda(p)=p^{n}+q_{n-2} p^{n-2}+\cdots+q_{0}\right\}
$$

with non-standard affine structure. (The LG potential $\lambda(p)$ usually is denoted as $W(p)$ ). The co-ordinates $t_{1}, \ldots, t_{n-1}$ on $M$ are determined from the system

$$
\partial_{t_{\alpha}} \lambda(p)=-\Phi_{\alpha}(p), \quad \alpha=1, \ldots, n-1
$$

for $\Phi_{1}(p), \ldots, \Phi_{n-1}(p)$ being the polynomials of degrees $0,1, \ldots, n-2$ orthogonal w.r.t. the scalar product

$$
\begin{gathered}
\langle\Phi, \Psi\rangle=\operatorname{res}_{p=\infty} \frac{\Phi(p) \Psi(p)}{d \lambda / d p} \\
\left\langle\Phi_{\alpha}, \Phi_{\beta}\right\rangle=\delta_{\alpha+\beta, n} .
\end{gathered}
$$

Then

$$
\eta_{\alpha \beta}=\left\langle\Phi_{\alpha}, \Phi_{\beta}\right\rangle, \quad C_{\alpha \beta \gamma}=\operatorname{res}_{p=\infty} \frac{\Phi_{\alpha} \Phi_{\beta} \Phi_{\gamma}}{d \lambda / d p}
$$

The genus zero chiral algebra coincides with truncated polynomials

$$
\Phi_{\alpha} \Phi_{\beta}=C_{\alpha \beta}^{\gamma}(t) \Phi_{\gamma} \quad(\bmod d \lambda / d p) .
$$

For generic $t$ the Frobenius algebra (0.27) is isomorphic to the trivial decomposable Frobenius algebra

$$
e_{\alpha} e_{\beta}=\delta_{\alpha \beta} e_{\alpha}, \quad\left\langle e_{\alpha}, e_{\beta}\right\rangle=\delta_{\alpha \beta}
$$

But for some special points $t$ in the coupling space (e.g., for $\lambda(p)=p^{n}, t=0$ ) the Frobenius algebra (0.27) is indecomposable. The partition function $F(t)$ was calculated by Krichever [10]. He showed that it coincides with $\log \tau(t)$, where $\tau(t)$ is the $\tau$-function for some particular solution of the averaged Gelfand-Dikii hierarchy

$$
\begin{gathered}
\partial_{t_{a}} L=\left[L,\left[L^{a / n}\right]_{+}\right], \quad a \neq k n, \\
L=\partial^{n}+q_{n-2} \partial^{n-2}+\ldots q_{0} .
\end{gathered}
$$

The averaging procedure is applied to the family $M$ of all constant solutions of $(0.29)$ (i.e. $m=0$ ).

In this paper we extend the LG formulae $[7,10]$ to the nonzero genus case via averaging of (0.29) over the family of $g$-gap solutions. We show that the averaged bi-hamiltonian structure of the averaged Gelfand-Dikii hierarchy is strongly involved in the $A_{n-1}$ - LG formalism for arbitrary genus $g$. More precisely, the coupling space is the variety of parameters of all $g$-gap solutions of (0.29). The Darboux coordinates for the first averaged Gelfand-Dikii P.B. [11] provide the affine structure in the coupling space $M$. The double-point correlator $(0.11)$ coincides with the metric on $M$ determining via (0.8) this averaged P.B. The correspondence $(0.16)$ is determined by the second averaged Gelfand-Dikii P.B. [11] (the averaged classical $W$-algebra). Also we calculate the genus $g$ partition function via $\tau$-function of the corresponding 
Whitham-type hierarchy. Because of the extension (0.4) new $2 g$ primary fields should be added for nonzero genus.

The paper consists of two sections. In the first one we describe the completion of the averaged GD hierarchy to obtain the corresponding Whitham-type herarchy. We describe bi-hamiltonian structure of the hierarchy and calculate the $\tau$-function for it. In the second section the developed formalism is applied to LG topological models of any genus $g$. All the proofs can be found in [2]. But some of the statements were not formulated in [2] explicitly.

\section{Bi-Hamiltonian Structure and Completion of the Averaged Gelfand-Dikii Hierarchy. $\tau$-Function of Whitham-Type Hierarchy}

The space $M=M_{g, n}$ of parameters of $g$-gap solutions of the GD hierarchy (0.29) coincides [12] with the moduli space of algebraic curves $C$ of genus $g$ with a marked point $Q_{\infty} \in C$ and with a meromorphic function $\lambda$ of degree $n$ (being equal to the order of $L$ ) with a pole only in $Q_{\infty}$. The dimension $N$ of the moduli space $M$ equals

$$
N=2 g+n-1 \text {. }
$$

If $P_{1}, \ldots, P_{N}$ are the branch points of $C$,

$$
\left.d \lambda\right|_{P_{\imath}}=0,
$$

then local co-ordinates on $M$ can be constructed as

$$
u^{i}=\lambda\left(P_{i}\right), \quad i=1, \ldots, N .
$$

The one-dimensional affine group $\lambda \mapsto \alpha \lambda+\beta, \alpha \neq 0$, acts on $M$ as

$$
u^{i} \mapsto \alpha u^{i}+\beta, \quad i=1, \ldots, N .
$$

Let $\tilde{M}$ be the covering of $M$ being obtained by fixation of a symplectic basis $a_{1}, \ldots, a_{g}, b_{1}, \ldots, b_{g} \in H_{1}(C, \mathbb{Z})$. Let $\mathscr{L} \tilde{M}$ be the loop space of the functions $u^{1}(x), \ldots, u^{N}(x)$ having their values in $M, x \in S^{1}$. The averaged GD-hierarchy on $\mathscr{L} \tilde{M}$ can be written in the Flaschka-Forest-McLaughlin (FFM) form [13]

$$
\partial_{T_{a}} d p=\partial_{X} d q^{(a)}, \quad a=1,2, \ldots
$$

Here $d q^{(a)}$ and $d p=d q^{(1)}$ are the normalized ${ }^{1}$ Abelian differentials of the second kind on $C$,

$$
\oint_{a_{\alpha}} d q^{(a)}=0, \quad \alpha=1, \ldots, g
$$

${ }^{1}$ Strictly speaking for the operators $L$ with real smooth coefficients the averaged hierarchy can be written in the FFM form with another normalization condition [14],

$$
\operatorname{Im} \oint_{\gamma} d q^{(a)}=0
$$

for any cycle $\gamma$ on $C$. Here we consider the averaged complexified GD-hierarchy being well-defined only after fixation of a basis in $H_{1}(C, \mathbb{Z})$ 
with the principal parts in $Q_{\infty}$ of the form

$$
d q^{(a)}=d \lambda^{a / n}+\text { regular terms }
$$

(a branch of $\lambda^{1 / n}$ for $\lambda \rightarrow \infty$ should be marked on $\tilde{M}$ ). Equivalently, (1.5) reads as

$$
\partial_{T_{a}} u^{i}=\left.\frac{d q^{(a)}}{d p}\right|_{P_{i}} \cdot u_{X}^{i}, \quad i=1, \ldots, N
$$

So the variables $u^{1}, \ldots, u^{N}$ are common Riemann invariants for the averaged GDhierarchy.

To describe the bi-hamiltonian structure of the hierarchy (1.5) let us define two metrics $d s^{2}$ and $d \tilde{s}^{2}$ on $\tilde{M}$ :

$$
\begin{gathered}
d s^{2}=\sum_{i=1}^{N} g_{\imath i}(u)\left(d u^{i}\right)^{2}, \quad d \tilde{s}^{2}=\sum_{i=1}^{N} \tilde{g}_{i i}(u)\left(d u^{\imath}\right)^{2}, \\
g_{i i}=\operatorname{res}_{P_{\imath}} \frac{(d p)^{2}}{d \lambda}, \quad \tilde{g}_{i i}=\operatorname{res}_{P_{\imath}} \frac{(d p)^{2}}{\lambda d \lambda}
\end{gathered}
$$

(cf. [15]).

Theorem 1. Both the metrics (1.9) are flat. The metric $d s^{2}$ is well defined and nondegenerate globally on $M$. The corresponding flat co-ordinates $t^{1}, \ldots, t^{N}$ for $d s^{2}$ have the form

$$
\begin{aligned}
t^{i} & =-n \underset{Q_{\infty}}{\operatorname{res}} \frac{\lambda^{(n-i) / n}}{n-i} d p, \quad i=1, \ldots, n-1, \\
t^{n-1+\alpha} & =\frac{1}{2 \pi i} \oint_{a_{\alpha}} p d \lambda, \\
t^{g+n-1+\alpha} & =\oint_{b_{\alpha}} d p, \quad \alpha=1, \ldots, g .
\end{aligned}
$$

These are well-defined globally on $\tilde{M}$. The metric $d s^{2}$ in the co-ordinates (1.11) has the form

$$
-n\left\langle d t^{i}, d t^{j}\right\rangle=\delta^{\imath+j, n}, \quad\left\langle d t^{n-1+\alpha}, d t^{g+n-1+\beta}\right\rangle=\delta^{\alpha \beta}
$$

otherwise zero.

Corollary. $\tilde{M}$ is an unramified covering over some domain in $\mathbb{C}^{N}$.

For genus zero the degree of this covering equals 1 .

The generators

$$
\partial=\sum_{i=1}^{N} \frac{\partial}{\partial u^{i}}, \quad D=\frac{1}{n} \sum_{i=1}^{N} u^{i} \frac{\partial}{\partial u^{i}}
$$


of the affine group (1.4) act on the co-ordinates (1.11) as follows:

$$
\begin{aligned}
\partial t^{i} & =0, & i \neq 1, & \\
\partial t^{1} & =-\frac{1}{n}, & & \\
D t^{i} & =(n-i+1) t^{i}, & i & =1, \ldots, n-1, \\
D t^{i} & =(n+1) t^{i}, & i & =n, \ldots, n+g-1, \\
D t^{i} & =t^{i}, & i & =n+g, \ldots, N .
\end{aligned}
$$

Remark. The variables $t^{1}, \ldots, t^{n-1}$ are the Casimirs for the first GD P.B., $t^{n}, \ldots$, $t^{g+n-1}$ are the action variables for the GD-hierarchy w.r.t. the first P.B. (see [16]) and $t^{g+n}, \ldots, t^{N}$ are the components of the wave number vector.

Since $\tilde{M}$ carries two flat metrics $d s^{2}$ and $d \tilde{s}^{2}$ the corresponding loop space $\mathscr{L} \tilde{M}$ carries two P.B. structures $\{,\}^{\sim}$ respectively (see Introduction above). It can be shown that $\{$,$\} coincides with the first averaged GD P.B. and \{,\} \sim$ coincides with the second averaged GD P.B. In the flat co-ordinates (1.11) the P.B. $\{$,$\} has the form$

$$
\left\{t^{\alpha}(X), t^{\beta}(Y)\right\}=\eta^{\alpha \beta} \delta^{\prime}(X-Y)
$$

for the $N \times N$-matrix $\left(\eta^{\alpha \beta}\right)$ being defined by (1.12).

Let $\tilde{M}$ be any Euclidean space with a scalar product $\eta^{\alpha \beta}$ in flat co-ordinates $t^{1}, \ldots, t^{N}$. Then the formula (1.15) determines a P.B. on $\mathscr{L} \tilde{M}$.

Theorem 2. Lagrangian planes $\mathscr{H} \subset \operatorname{Funct}(\mathscr{L} \tilde{M})$ of functionals of the form

$$
\left.\mathscr{H}=\int h(t(X)) d X\right\}
$$

are in 1-1 correspondence with curvilinear orthogonal co-ordinate systems in $\tilde{M}$.

Remark. $\mathscr{B} \tilde{M}$ is not a symplectic manifold due to degeneracy of P.B. (1.15). Nevertheless Lagrangian planes can be defined as maximal isotropic subspaces of functionals Funct $(\mathscr{L} \tilde{M})$ on $\mathscr{L} \tilde{M}$.

Theorem 2 is a reformulation of Tsarev's results [17]. For given curvilinear orthogonal co-ordinates $u^{1}, \ldots, u^{N}$ in $\tilde{M}$ the densities $h$ of the functionals $H \in \mathscr{H}$ are determined from the diagonality of the covariant Hessian

$$
\nabla_{u^{i}} \nabla_{u^{j}} h(u)=0, \quad i \neq j
$$

For the moduli space $\tilde{M}=\tilde{M}_{g, n}$ we have a canonical curvilinear orthogonal local co-ordinate system $u^{1}, \ldots, u^{N}$ (the branch points of the Riemann surface $C \stackrel{\lambda}{\longrightarrow} \mathbb{C}$ ). Corollary. The loop space $\mathscr{L} \tilde{M}$ of the moduli space $\tilde{M}=\tilde{M}_{g, n}$ carries a canonical Lagrangian plane $\mathscr{H} \subset \operatorname{Funct}(\mathscr{L} \tilde{M})$ of the form (1.16).

Definition. The commuting family of Hamiltonian systems

$$
\partial_{T} u^{i}=\left\{u^{i}(X), H\right\}, \quad H \in \mathscr{H}
$$

is called the Whitham-type hierarchy on $\mathscr{L} \tilde{M}$.

We shall show that this is an extension (and, therefore, a completion) of the averaged GD hierarchy (1.5). 
Theorem 3. 1) The functionals with densities $h(u), u \in \tilde{M}$, of the form

$$
\begin{gathered}
h(u)=\operatorname{res}_{Q_{\infty}} \lambda^{k / n} d p, \quad k \neq l n, \\
h(u)=\frac{1}{2 \pi i} \oint_{a_{\alpha}} \lambda^{k} d p, \quad h(u)=\oint_{b_{\alpha}} \lambda^{k-1} d p, \quad \alpha=1, \ldots, g, \quad k=1,2, \ldots
\end{gathered}
$$

span the canonical Lagrangian plane $\mathscr{H} \subset \operatorname{Funct}(\mathscr{B} \tilde{M})$.

2) The P.B. $\{$,$\} and \{,\}^{\sim}$ are compatible (i.e. any their linear combination again gives a P.B.).

3) The equations of Whitham-type hierarchy (1.18) are bi-hamiltonian with respect to $\{$,$\} and \{,\}^{\sim}$.

To describe FFM-representations for the Whitham-type hierarchy (1.18) let us consider the standard fiber bundle

$$
\underset{\tilde{M}}{\downarrow} C
$$

(the fiber over $u \in \tilde{M}$ is the curve $C=C(u)$ with marked homology basis and a point $Q_{\infty}$ and a function $\lambda$ ). This has a canonical connection: the curves $\lambda=$ const are horizontal by definition. Let us define multivalued Abelian differentials on $C$ as Abelian differentials $\Omega=\Omega(P)$ on the universal covering of $C$ such that

$$
\Delta_{\gamma} \Omega(P) \equiv \Omega(P+\gamma)-\Omega(P)=\sum_{k} C_{k}(\gamma) \lambda^{k} d \lambda
$$

for any cycle $\lambda \in H_{1}(C, \mathbb{Z})$. A family $\Omega=\Omega(P, u)$ of multivalued Abelian differentials on the curve $C=C(u)$ smoothly depending on $u \in \tilde{M}$ is called horizontal if:

1) It is holomorphic for any $u$ on $C \backslash Q_{\infty}$.

2) Its covariant derivatives $\partial_{u^{j}} \Omega$ are Abelian differentials of the second kind on $C$ (i.e. with zero residues) with double poles only in the branch points $P_{1}, \ldots, P_{N}$ and with zero $a$-periods.

Let $D(\tilde{M})$ be the quotient of the space of all horizontal differentials over the subspace of differentials of the form

$$
\sum_{k} c_{k} \lambda^{k} d \lambda
$$

Proposition. The space $D(\tilde{M})$ is spanned by the following horizontal differentials:

$$
\Omega^{(k)}=-\frac{1}{k} d q^{(k)}, \quad k=1,2, \ldots, k \neq l n
$$

2) holomorphic differentials $\omega_{\alpha} \equiv \omega_{\alpha}^{(0)}$

$$
\oint_{a_{l}} \omega_{k}=2 \pi i \delta_{k l} ;
$$

3) multivalued normalized (i.e. with zero a-periods) holomorphic on $C$ differentials $\sigma_{\alpha}^{(k)}, k=1,2, \ldots, \alpha=1, \ldots, g$ with the increments of the form

$$
\Delta_{b_{\alpha}} \sigma_{\alpha}^{(k)}=-d\left(\lambda^{k}\right)
$$

other increments vanish; 
4) multivalued normalized holomorphic on $C$ differentials $\omega_{\alpha}^{(k)}, \alpha=1, \ldots, g$, $k=1,2, \ldots$ with increments of the form

$$
\Delta_{a_{\alpha}} \omega_{\alpha}^{(k)}=d\left(\lambda^{k}\right)
$$

Let us define a pairing

$$
\Omega, \Omega^{\prime} \mapsto V_{\Omega \Omega^{\prime}}
$$

for any two horizontal differentials $\Omega, \Omega^{\prime}$ via the formula

$$
\begin{aligned}
V_{\Omega \Omega^{\prime}}= & \operatorname{res}\left[\left(\int_{Q_{\infty}} \Omega\right)_{+} \Omega^{\prime}\right] \\
& +\sum_{\alpha=1}^{g}\left[\oint_{a_{\alpha}} \Omega \oint_{b_{\alpha}} \Omega^{\prime}-\frac{1}{2 \pi i} \oint_{a_{\alpha}}\left(d^{-1} \Delta_{b_{\alpha}} \Omega\right) \Omega^{\prime}\right. \\
& \left.+\frac{1}{2 \pi i} \oint_{b_{\alpha}}\left(d^{-1} \Delta_{a_{\alpha}} \Omega\right) \Omega^{\prime}\right] .
\end{aligned}
$$

Here $\left(\int \Omega\right)_{+}$means the principal part near $Q_{\infty}$ of the meromorphic function $\int \Omega$. For horizontal differentials the function $V_{\Omega \Omega^{\prime}}=V_{\Omega \Omega^{\prime}}(u)$ on $\tilde{M}$ is well-defined and symmetric up to an additive constant. The main property of it is in the identity

$$
\partial_{u^{\jmath}} V_{\Omega \Omega^{\prime}}(u)=\operatorname{res}_{P_{j}} \frac{\Omega \Omega^{\prime}}{d \lambda}, \quad j=1, \ldots, N .
$$

Theorem 4. The map $D(\tilde{M}) \rightarrow \mathscr{H} /$ const of the form

$$
\Omega \mapsto V_{d p, \Omega}
$$

is a linear isomorphism of the space of horizontal differentials onto the quotient of the canonical Lagrangian plane over constants. The inverse map $\mathscr{H} \rightarrow D(M)$,

$$
\mathscr{H} \ni H=\int h(u) d X \mapsto \Omega_{h} \in D(M)
$$

is determined by the property

$$
\{d p, H\}^{\sim}=\left(q_{h}-\frac{1}{n}\right) \partial_{X} \Omega_{h}, \quad h(c u)=c^{q_{h}} h(u) .
$$

Equivalently, the skew-gradient of $H=\int h(u) d X$ w.r.t. $\{,\}^{\sim}$ can be represented in the FFM form

$$
\partial_{T} d p=\left(q_{h}-\frac{1}{n}\right) \partial_{X} \Omega_{h}, \quad h(u) \in \mathscr{H} .
$$

Remark. The Hamiltonian of the flow (1.33) w.r.t. $\{$,$\} equals const \cdot \partial^{-1} h$.

For densities $h(u)$ of the form (1.19a), Eqs. (1-33) coincide with the averaged GD-hierarchy (1.5). The densities $h(u)$ of the form (1.19b) provide the extension of (1.5) to obtain a complete hierarchy.

Lemma. For any two functions $f(u), g(u) \in \mathscr{B}$ the following identity holds:

$$
\sum \underset{d \lambda=0}{\operatorname{res}} \frac{\Omega_{f} \Omega_{g}}{d \lambda}=\langle d f, d g\rangle .
$$

Here $\langle$,$\rangle means the scalar product w.r.t. the metric d s^{2}$.

Note that the both P.B. $\{$,$\} and \{,\}^{\sim}$ are involved in the identity (1.34). 
Let us choose some numeration $h_{A}(u), a=1,2, \ldots$ of the basis $(1.19 \mathrm{a}, \mathrm{b})$ such that

$$
\Omega_{h_{1}}=d p
$$

(so $h_{1}=t_{1}$ ). The Whitham-type hierarchy has the form

$$
\partial_{T_{A}} d p=\partial_{X} \Omega_{A}, \quad A=1,2, \ldots, \quad \Omega_{A} \equiv \Omega_{h_{A}} .
$$

Any horizontal differential $\Omega$ determines a solution $u^{i}=u^{i}\left(X=T_{1}, T_{2}, \ldots\right)$. $i=1, \ldots, N$ of the hierarchy (1.36) via the Tsarev-Krichever procedure $[17,14]$

$$
\left.\left(\sum T_{A} \Omega_{A}+\Omega\right)\right|_{d \lambda=0}=0
$$

(this is a system of $N$ equations for $N$ unknown functions $u^{i}(T)$ ).

Theorem 5. The formula (1.37) locally gives general solution of the hierarchy (1.36) being analytic in all the times $T_{A}$.

The corresponding $\tau$-function $\tau=\tau_{\Omega}(T)$ of the Whithman-type (1.36) has the form $^{2}$

$$
\log \tau_{\Omega}(T)=-\frac{1}{2} V_{\Omega^{\prime}, \Omega^{\prime}}
$$

for

$$
\Omega^{\prime}=\Omega+\sum t_{A} \Omega_{A}
$$

It is well defined up to multiplication by exponent of a quadratic form in $T_{A}$-variables with constant coefficients. The main property of the $\tau$-function of the Whitham-type hierarchy reads as

$$
\partial_{t_{A}} \partial_{t_{B}} \log \tau_{\Omega}=-V_{\Omega_{A} \Omega_{B}}
$$

Remark. The original averaged GD hierarchy (1.5) has an additional feature: it has a class of exact solutions that can be $C^{1}$-extended onto

These solutions have the form

$$
\tilde{M}_{n}=\bigcup_{g} \tilde{M}_{g, n}
$$

$$
\left.\left(\sum T_{a} d q^{(a)}+\Omega\right)\right|_{d \lambda=0}=0
$$

for any normalized differential $\Omega$ of the second kind with pole only in $Q_{\infty}$. This point can be used for analytic solution of Novikov's problem (see [4]) of multivalued functions evolution in the dispersive hydrodynamics.

\section{Topological Landau-Ginsburg Models of Genus $g$}

Here we give explicit formulae for the partition function (at the tree level) and for the chiral ring of $A_{n-1}$-LG topological model for any genus $g$.

1) Coupling space is the moduli space $\tilde{M}=\tilde{M}_{g, n}$.

2) Flat co-ordinates $t^{1}, \ldots, t^{N}$ on $\tilde{M}$ have the form (1.11) (they are the flat coordinates for the metric $d s^{2}$ and, therefore, the densities of Casimirs for $\{$,$\} ).$

3) The corresponding primary fields $\Phi_{1}=d p, \Phi_{2}, \ldots, \Phi_{N}$ have the form

$$
\Phi_{\alpha}=\eta_{\alpha \beta} \Omega_{t^{\beta}}
$$

(we recall that the map $f \mapsto \Omega_{f}$ was described above via the second P.B. $\{,\}^{\sim}$ ).

\footnotetext{
${ }^{2}$ For genus zero the $\tau$-function of Whitham hierarchy (i.e. of dispersionless Lax equations) was constructed by Krichever [10]
} 
More explicitly:

$$
\begin{gathered}
\Phi_{i}=-n \Omega^{(i)}, \quad i=1, \ldots, n-1, \\
\Phi_{n-1+\alpha}=\omega_{\alpha}, \quad \Phi_{g+n-1+\alpha}=\sigma_{\alpha}, \quad \alpha=1, \ldots, g,
\end{gathered}
$$

where $\omega_{\alpha}, \sigma_{\alpha}=\sigma_{\alpha}^{(1)}$ are defined by (1.24), (1.25).

4) LG potential is $\lambda=\lambda(p)$, where $p=\int d p$. In other words

$$
\partial_{t^{\alpha}}(\lambda d p)_{p=\text { const }}=-\Phi_{\alpha} .
$$

5) The correlation functions of the primary fields have the form

$$
\begin{gathered}
\left\langle\Phi_{\alpha} \Phi_{\beta}\right\rangle=\eta_{\alpha \beta}=\sum \underset{d \lambda=0}{\operatorname{res}} \frac{\Phi_{\alpha} \Phi_{\beta}}{d \lambda} \\
\left\langle\Phi_{\alpha_{1}} \ldots \Phi_{\alpha_{k+2}}\right\rangle=\sum \operatorname{res} \frac{\Phi_{\alpha_{1}} \ldots \Phi_{\alpha_{k+2}}}{d \lambda(d p)^{k}} .
\end{gathered}
$$

The matrix $\eta_{\alpha \beta}$ is of the form (1.12) [this follows from the identity (1.34)].

6) The chiral algebra $C_{\alpha \beta}^{\gamma}(t)$ has the form

$$
\Phi_{\alpha} \Phi_{\beta}=C_{\alpha \beta}^{\gamma} \Phi_{\gamma} d p \quad(\bmod d \lambda \cdot D(\tilde{M})) .
$$

This is a Frobenius algebra for any $t$ with the constant invariant scalar product (2.4) and with the unit $\Phi_{1}=d p$.

7) The partition function $F=\log \tau$ has the form

$$
F=-\frac{1}{2} V_{p d \lambda, p d \lambda} \text {. }
$$

Since the differential $p d \lambda$ is not a horizontal one the last formula should be clarified. We have the identity

$$
p d \lambda=n \Omega^{(n+1)}+\sum_{\alpha=1}^{N} t^{\alpha} \Phi_{\alpha}
$$

So

$$
V_{p d \lambda, p d \lambda}=n^{2} V_{\Omega^{(n+1)}, \Omega^{(n+1)}}+2 n \sum_{\alpha=1}^{N} t^{\alpha} V_{\Omega^{(n+1)}, \Phi_{\alpha}}+\sum_{\alpha, \beta=1}^{N} t^{\alpha} t^{\beta} V_{\Phi_{\alpha} \Phi_{\beta}} .
$$

The dependence of the coefficients in the formula (2.9) on $t$ is determined from (1.11) [or, equivalently, from the vanishing of the right-hand side of $(2.8)$ in the branch points $d \lambda=0$-cf. (1.37) above].

The partition function $F$ posesses a quasihomogeneous property of the form

$$
\begin{aligned}
& F\left(c^{n} t^{1}, \ldots, c^{2} t^{n-1}, c^{n+1} t^{n}, \ldots, c^{n+1} t^{g+n-1}, c t^{g+n}, \ldots, c t^{N}\right) \\
& \quad=c^{2(n+1)} F(t) .
\end{aligned}
$$

Obviously the fields $\Phi_{1}, \ldots, \Phi_{n-1}$ are lifted from the genus zero case. But the $2 g$ fields $\Phi_{n}, \ldots, \Phi_{N}$ have no good zero genus limit.

Remark. The Hessian

$$
\left(\partial_{t^{\alpha}} \partial_{t^{\beta}} F(t)\right)_{n \leq \alpha, \beta \leq g+n-1}
$$

coincides with the period matrix of the algebraic curve $C$ :

$$
\tau_{i j}=\oint_{b_{i}} \omega_{j}
$$


It would be very interesting to find the Virasoro-type constraints uniquely characterizing the partition function (2.7) (for genus zero some constraints were considered in [10]). This could give a new approach in the solution of the Schottky problem of specifying period matrices of Riemann surfaces [19].

\section{Appendix. Geometry and Deformations of Frobenius Algebras}

Let $A$ be any $N$-dimensional (commutative) Frobenius algebra and $M=A^{*}$ (the dual space). A multiplication is defined on $T^{*} M$ : if $u^{1}, \ldots, u^{N}$ is a basis in $A$ (providing the co-ordinate system in $M$ ) then

$$
d u^{i} \cdot d u^{k}=c_{k}^{i j} d u^{k}
$$

$c_{k}^{i j}$ being the structure constants of $A$. The non-degenerate scalar product on $T^{*} M$ (and, therefore, a metric on $M$ ) is defined by the formula

$$
\langle d f, d g\rangle=2 i_{D}(d f \cdot d g)
$$

$D=u^{i} \frac{\partial}{\partial u^{2}}$ is the dilation generator. It was shown in [9] that the metric (A.2) is flat and the corresponding Levi-Civitá connection has the form

$$
\nabla^{i} T^{j}=\partial^{\imath} T^{j}-c_{s}^{i j} T^{s}
$$

(raising of indexes using the metric (A.2)). The flat co-ordinates $t^{1}, \ldots, t^{N}$ can be introduced via an appropriate quadratic substitution

$$
\begin{gathered}
u^{i}=\frac{1}{2} a_{\alpha \beta}^{i} t^{\alpha} t^{\beta} \\
\left\langle d t^{\alpha}, d t^{\beta}\right\rangle=\eta^{\alpha \beta}=\text { const . }
\end{gathered}
$$

Let us consider the coefficients

$$
c^{i_{1}, \ldots, i_{n}}=2 c_{s_{1}}^{i_{1} i_{2}} c_{s_{2}}^{s_{1} i_{3}} \ldots c_{s_{n-1}}^{s_{n-2} i_{n}} u^{s_{n-1}}
$$

and the functions

$$
\tilde{c}_{\gamma}^{\alpha \beta}(t)=\frac{\partial t^{\alpha}}{\partial u^{\imath}} \frac{\partial t^{\beta}}{\partial u^{j}} \frac{\partial u^{k}}{\partial t^{\gamma}} c_{k}^{\imath j} .
$$

Proposition. The functions (A.7) determine a deformation of the Frobenius algebra $A$ with constant invariant scalar product $\left(\eta^{\alpha \beta}\right)$. The deformation can be represented in the form (0.20) for the "partition function"

$$
F(t)=\sum_{n=3}^{\infty} \frac{2^{n-1}}{n} c^{i_{1} \ldots i_{n}} u_{i_{1}} \ldots u_{i_{n}} .
$$

Proof. It is sufficient to prove that in the curvilinear co-ordinates $u^{1}, \ldots, u^{N}$ the function (A.8) satisfies the equation

$$
\nabla^{i} \nabla^{j} \nabla^{k} F=c^{\imath j k}
$$

The proof of (A.9) is straightforward using the identities

$$
\nabla^{l} c_{k}^{i j}=-c_{s}^{i j} c_{k}^{s l}, \quad \nabla^{k} u_{\imath}=\frac{1}{2} \delta_{i}^{k}
$$


Notes added in proof. 1. After this paper was finished the author was shown a paper [20] of Krichever. In this paper the averaged $G D$ hierarchy for genus $g>0$ also was considered from the point of view of topological field theory. But no extension of the averaged $G D$ hierarchy was considered. Without such an extension for $g>0$ it is impossible to construct a closed primary operator algebra. 2. As it was argued in [21] the models of the present paper (for $g>0$ ) can be obtained from $A_{n-1}$ minimal models as a result of "phase transition". The integrability of the nonlinear system for the partition function being imposed by associativity of the Frobenius algebra $(0.19)-(0.21),(0.14)$, also is proved in [21]. The hierarchy of systems of hydrodynamic type of the form (1.18) proves to describe coupling to topological gravity.

20. Krichever, I.: Topological minimal models and soliton equations. Talk on the 1st A. Sakharov Congress, Moscow, May 1991

21. Dubrovin, B.: Integrable systems in topological field theory. Preprint INFN-NA-IV-91/26, December 1991. Submitted to Nucl. Phys. B

\section{References}

1. Whitham, G.B.: Linear and nonlinear waves. New York: Wiley 1974

Dobrokhotov, S., Maslov, V.: Soviet Sci. Rev., Math. Phys. Rev. 3, 221 (1982), OPA, Amsterdam

2. Dubrovin, B.: Differential geometry of moduli spaces and its applications to soliton equations and to topological conformal field theory. Preprint n. 117 of Scuola Normale Superiore, Pisa, November 1991

3. Dubrovin, B., Novikov, S.: Sov. Math. Doklady 27, 665 (1983)

4. Dubrovin, B., Novikov, S.: Russ. Math. Surv. 44:6, 35 (1989)

5. Eguchi, T., Yang, S.-K.: $N=2$ superconformal models as topological field theories. Tokyo preprint UT-564

Witten, E.: Commun. Math. Phys. 118, 411 (1988)

Vafa, C., Warner, N.P.: Phys. Lett. B 218, 51 (1989)

Lerche, W., Vafa, C., Warner, N.P.: Nucl. Phys. B 324, 427 (1989)

6. Curtis, C., Reiner, I.: Representation theory of finite groups and associative algebras. New York: Interscience 1962

7. Dijkgraaf, R., Verlinde, E., Verlinde, H.: Topological strings in $d<1$. Princeton preprint PUPT1204, IASSNS-HEP-90/71

8. Vafa, C.: Topological Landau-Ginsburg models. Preprint HUTP-90/AO64

Verlinde, E., Verlinde, H.: A solution of two-dimensional topological gravity. Preprint IASSNSHEP-90/45

9. Balinski, A., Novikov, S.: Sov. Math. Doklady 32, 228 (1985)

10. Krichever, I.: The dispersionless Lax equations and topological minimal models. Preprint ISI Torino, April 1991 (to appear in Commun. Math. Phys. 1991)

11. Gelfand, I., Dikii, L.: A family of Hamilton structures related to integrable systems. Preprint IPM/136 (1978) (in Russian)

Gelfand, I., Dorfman, I.: Funct. Anal. Appl. 14, 223 (1980)

Adler, M.: Invent. Math. 50, 219 (1979)

12. Krichever, I.: Funct. Anal. Appl. 11, 15 (1977)

13. Flaschka, H., Forest, M.G., McLaughlin, D.W.: Commun. Pure Appl. Math. 33, 739 (1980)

14. Krichever, I. Funct. Anal. Appl. 22, 206 (1988)

15. Dubrovin, B.: Funct. Anal. Appl. 24 (1990)

16. Veselov, A., Novikov, S.: Sov. Math. Doklady (1982)

17. Tsarev, S.: Math. USSR Izvestiya (1990)

18. Blok, B., Varchenko, A.: Topological conformal field theories and the flat co-ordinates. Preprint IASSNS-HEP-91/5

19. Dubrovin, B.: Russ. Math. Surv. 36:2 (1981); Dubrovin, B: Math. USSR Izvestiya 19:2 (1981)

Shiota, T.: Inv. Math. 83, 333 (1986)

Arbarello, E., De Concini, C.: Ann. Math. 120, 119 (1984) 
